\title{
GENERALIZED URN SCHEMES AND TECHNOLOGICAL DYNAMICS
}

\author{
Giovanni Dosi \\ University of Rome 'La Sapienza', Rome, Italy \\ and \\ International Institute for Applied Systems Analysis \\ Laxenburg, Austria \\ Yuri Ermoliev and Yuri Kaniovski \\ International Institute for Applied Systems Analysis \\ Laxenburg, Austria
}

RR-94-11

September 1994

Reprinted from Journal of Mathematical Economics 23(1994) 1-19.

INTERNATIONAL INSTITUTE FOR APPLIED SYSTEMS ANALYSIS

Laxenburg, Austria 
Research Reports, which record research conducted at IIASA, are independently reviewed before publication. Views or opinions expressed herein do not necessarily represent those of the Institute, its National Member Organizations, or other organizations supporting the work.

Reprinted with permission from The Journal of Mathematical Economics 23(1994) 1-19. Copyright @ 1994 Elsevier Science BV.

All rights reserved. No part of this publication may be reproduced or transmitted in any form or by any means, electronic or mechanical, including photocopy, recording, or any information storage or retrieval system, without permission in writing from the copyright holder.

Printed by Novographic, Vienna, Austria. 


\section{Systems Analysis of Technological and Economic Dynamics}

This new research project at IIASA is concerned with modeling technological and organizational change; the broader economic developments that are associated with technological change, both as cause and effect; the processes by which economic agents - first of all, business firms - acquire and develop the capabilities to generate, imitate, and adopt technological and organizational innovations; and the aggregate dynamics - at the levels of single industries and whole economies - engendered by the interactions among agents which are heterogeneous in their innovative abilities, behavioral rules and expectations. The central purpose is to develop stronger theory and better modeling techniques. However, the basic philosophy is that such theoretical and modeling work is most fruitful when attention is paid to the known empirical details of the phenomena the work aims to address: therefore, a considerable effort is put into a better understanding of the 'stylized facts' concerning corporate organization routines and strategy; industrial evolution and the 'demography' of firms; patterns of macroeconomic growth and trade.

From a modeling perspective, over the last decade considerable progress has been made on various techniques of dynamic modeling. Some of this work has employed ordinary differential and difference equations, and some of it stochastic equations. A number of efforts have taken advantage of the growing power of simulation techniques. Others have employed more traditional mathematics. As a result of this theoretical work, the toolkit for modeling technological and economic dynamics is significantly richer than it was a decade ago.

During the same period, there have been major advances in the empirical understanding. There are now many more detailed technological histories available. Much more is known about the similarities and differences of technical advance in different fields and industries and there is some understanding of the key variables that lie behind those differences. A number of studies have provided rich information about how industry structure coevolves with technology. In addition to empirical work at the technology or sector level, the last decade has also seen a great deal of empirical research on productivity growth and measured technical advance at the level of whole economies. A considerable body of empirical research now exists on the facts that seem associated with different rates of productivity growth across the range of nations, with the dynamics of convergence and divergence 
in the levels and rates of growth of income in different countries, with the diverse national institutional arrangements in which technological change is embedded.

As a result of this recent empirical work, the questions that successful theory and useful modeling techniques ought to address now are much more clearly defined. The theoretical work described above often has been undertaken in appreciation of certain stylized facts that needed to be explained. The list of these 'facts' is indeed very long, ranging from the microeconomic evidence concerning for example dynamic increasing returns in learning activities or the persistence of particular sets of problem-solving routines within business firms; the industry-level evidence on entry, exit and size-distributions - approximately log-normal; all the way to the evidence regarding the time-series properties of major economic aggregates. However, the connection between the theoretical work and the empirical phenomena has so far not been very close. The philosophy of this project is that the chances of developing powerful new theory and useful new analytical techniques can be greatly enhanced by performing the work in an environment where scholars who understand the empirical phenomena provide questions and challenges for the theorists and their work.

In particular, the project is meant to pursue an 'evolutionary' interpretation of technological and economic dynamics modeling, first, the processes by which individual agents and organizations learn, search, and adapt; second, the economic analogs of 'natural selection' by which interactive environments - often markets - winnow out a population whose members have different attributes and behavioral traits; and, third, the collective emergence of statistical patterns, regularities, and higher-level structures as the aggregate outcomes of the two former processes.

Together with a group of researchers located permanently at IIASA, the project coordinates multiple research efforts undertaken in several institutions around the world, organizes workshops and provides a venue of scientific discussion among scholars working on evolutionary modeling, computer simulation and non-linear dynamical systems.

The research will focus upon the following three major areas:

1. Learning Processes and Organizational Competence.

2. Technological and Industrial Dynamics

3. Innovation, Competition, and Macrodynamics 


\section{Preface}

Since January 1994 the Systems Analysis of Technological and Economic Dynamics Project (TED) has been continuing the chain of studies on technological dynamics at IIASA that can be traced as far back as the early 1980s.

This paper deals with a class of stochastic models of technological diffusion based on so-called generalized urn schemes. The models exhibit nonergodic limit behavior. In contrast to existing models based on urn schemes, the initial state influences not only the shape of the limit distribution, but also the support of this distribution. The authors consider the case of strategic price manipulation that can be applied to other cases, for example, models of bargaining.

It represents a fragment of the study on one of the three major directions of the TED project, namely, modeling of macroeconomic issues.

Peter E. de Jánosi

Director 


\title{
Generalized urn schemes and technological dynamics*
}

\section{Giovanni Dosi}

University of Rome 'La Sapienza', Rome, Italy

\section{Yuri Ermoliev}

International Institute for Applied Systems Analysis, Laxenburg, Austria

\author{
Yuri Kaniovski \\ International Institute for Applied Systems Analysis, Laxenburg, Austria
}

Submitted August 1991, accepted February 1993

Adaptive (path dependent) processes of growth modeled through urn schemes find important applications to economic dynamics (and also to other disciplines, such as biology, physics, chemistry). The paper presents some further properties of generalized urn schemes and studies dynamic stochastic processes characterized by both positive and, possibly, negative feedbacks of a functional form as 'badly behaved' as possible. Two applications to technological diffusion are considered.

One of the models tackles the case when there is a separation within the pool of adopters which can be interpreted as the outcome of adaptive learning on the features of the new technologies by imperfectly informed agents. Other examples deal with dependence of final market shares of two technologies on the pricing policies of the firms which produce them. The stochasticity of the processes is caused by some mixed strategies used by the adopters or/and imperfectness of the information which they possess.

Key words: Urn schemes; Technological dynamics

\section{Introduction}

The competition among new technologies is clearly a fundamental aspect

Correspondence to: Giovanni Dosi, Department of Economics, University of Rome 'La Sapienza', via Nomentana, 41, 00169 Rome, Italy.

*The authors gratefully acknowledge the support received from the Italian Research Council (CNR - 'Altri interventi'), from the Italian Ministry for the Universities and for Scientific and Technological Research (MURST - Ricerche $40 \%$ ) and from IIASA. They also wish to thank Kenneth Arrow, an anonymous referee and the editors for their useful comments. The usual caveats apply. 
of the process of growth and transformation of contemporary economies. So also is the competition among different firms which embody different technologies, different expectations and, possibly, show different market behaviors. In turn, it is increasingly acknowledged that technological innovations are likely to involve some forms of dynamic increasing returns, i.e., some positive feedbacks along their diffusion trajectories [cf. Dosi et al. (1988) and Anderson et al. (1988); for an interpretation of the empirical evidence, Dosi (1988)]. To study the underlying competitive process a wide variety of mathematical approaches has been suggested within and outside economic analysis, e.g., ordinary differential equations [as in Polterovich and Henkin (1988)], in particular with trajectories on the unit simplex, i.e., of the population type [as in Silverberg et al. (1988)], and generalized urn schemes which generate stochastic dynamic systems with discrete time and trajectories from the unit simplex [cf. Arthur (1988) and Arthur et al. (1983, 1987c)]. In the following we shall present some extensions of the latter approach able to handle positive feedbacks that are only 'local' - in the sense that they occur only under particular states on the diffusion trajectory - or the coexistence of both positive and negative feedback mechanisms in the competitive process (the simplest variants of the models presented here can be formally reduced to the ones considered in earlier works [Arthur (1988), Arthur et al. (1983, 1987c)]. We shall apply these generalized urn schemes to two topics concerning competing technologies.

As a first application, we study the dependence of limit market shares from some mixed strategies used by risk-averse adopters of the technologies. Conceptually, these mixed strategies, which generate some separation within the pool of adopters, can be interpreted as the outcome of adaptive learning on the features of the new technologies by imperfectly informed agents. ${ }^{1}$ For example, one may assume that the latter decide by observing the choice of previous adopters and 'trusting' them, to different degrees. Such a behavior is also easily interpretable in terms of 'bounded rationality', and/or some interdependence in the returns of individual adopters, depending on the relative frequencies of the chosen technologies. Economic examples of such interdependences are particular clear with respect to innovation and innovation diffusion [cf. Arthur (1988), David (1985, 1993), Dosi (1988), Hanson (1985)] whereby dynamic increasing returns and various sorts of externalities are generally observed. However, the modeling techniques suggested here could be in principle applied, with the proper modifications, to other economic domains involving interdependence of expectations [such as those discussed in e.g., Frydman (1982) and Frydman and Phelps (1983)] and speculation [cf. Kirman (1991)].

The second application concerns the dependence of the final market shares

\footnotetext{
${ }^{1} \mathrm{~A}$ similar problem, in the case of Bayesian learning, is analyzed in Arthur and Lane (1993).
} 
of two technologies on the pricing policies of the firms which produce them. In the following, we suppose that each of the firms decreases its price until it reaches a certain market share (measured by the proportion of units of the technology they produce among all units of all technologies which have been sold up to that time). Above that share, prices are increased. An economic interpretation of such a behavioral hypothesis is that firms - as often found in the business literature - follow strategies aimed at market pre-emption and at learning economies until they reach a dominant market position which they can exploit thereafter. Whether such pricing policies can be derived from strategies of intertemporal profit maximization under imperfect information or not is by no means essential to the model. In principle, it is meant to analyze the share dynamics of different technologies with endogenous prices (no matter whether the latter are microfounded on intertemporally optimizing agents or not). At each time, prices of each technology can be different but adopters may not instantly switch from one to another due to e.g., imperfect information, network externalities, etc. Indeed, the stochasticity of the process is caused by some mixed strategies used by the adopters in the case of approximately equal prices on competing technologies.

Well beyond the two specifications of the model which we are going to present here, one of our aims is to illustrate the general applicability to economic and technological dynamics of generalized urn schemes which generate discrete time stochastic dynamic systems with multiple equilibria. Some of them turn out to be attainable (i.e., they realize with positive probabilities). They represent those limit proportions of competing technologies which are feasible. In the following we shall use known results [Arthur et al. (1987a, b, 1988), Hill et al. (1980)] concerning generalized urn schemes and suggest some further developments.

In section 2 we shall present the basic theorems on generalized urn schemes. Section 3 studies technological competition with imperfect information ard endogenous preferences for the two technologies. In section 4 we analyze the case with endogenous prices.

\section{The theory of generalized urn schemes}

Think of an urn of infinite capacity with black and white balls. Starting with $n_{\mathrm{w}} \geqq 1$ white balls and $n_{\mathrm{b}} \geqq 1$ black balls in the urn, a ball is added at time instants $t=1,2 \ldots$. It will be white with probability $f\left(X_{t}\right)$ and black with probability $1-f\left(X_{t}\right)$. Here $f(\cdot)$ is a function [it is sometimes called an urn function [Hill et al. (1980)]], which maps $R(0,1)$ into $[0,1][R(0,1)$ stands for the set of rational numbers from $(0,1)]$. We call $X_{t}$ the proportion of white balls in the urn at time $t$. Then the dynamics of $X_{t}$ is given by the relation

$$
X_{t+1}=X_{t}+\left(t+n_{\mathrm{w}}+n_{\mathrm{b}}\right)^{-1}\left[\xi_{t}\left(X_{t}\right)-X_{t}\right], \quad t \geqq 1, \quad X_{1}=n_{\mathrm{w}}\left(n_{\mathrm{w}}+n_{\mathrm{b}}\right)^{-1} .
$$


Here $\xi_{t}(x), t \geqq 1$, are random variables independent in $t$ such that

$$
\xi_{t}(x)= \begin{cases}1 & \text { with probability } f(x), \\ 0 & \text { with probability } 1-f(x)\end{cases}
$$

Designate $\xi_{t}(x)-\mathrm{E} \xi_{t}(x)=\xi_{t}(x)-f(x)$ by $\zeta_{t}(x)$. Then we have

$$
\begin{gathered}
X_{t+1}=X_{t}+\left(t+n_{\mathrm{w}}+n_{\mathrm{b}}\right)^{-1}\left\{\left[f\left(X_{t}\right)-X_{t}\right]+\zeta_{t}\left(X_{t}\right\},\right. \\
t \geqq 1, \quad X_{1}=n_{\mathrm{w}}\left(n_{\mathrm{w}}+n_{\mathrm{b}}\right)^{-1} .
\end{gathered}
$$

Due to $\mathrm{E} \zeta(x)=0$, given $X_{t}=x$, the system (1) shifts on average at time $t \geqq 1$ on the value $\left(t+n_{\mathrm{w}}+n_{\mathrm{b}}\right)^{-1}[f(x)-x]$. Consequently limit points of the sequence $\left\{X_{t}\right\}$ have to belong to the set $B$ of zeros of the function $f(x)-x$ (for $x \in[0,1]$ ). The following statement confirms this hypothesis.

Theorem 1. [Arthur et al. (1987b)]. The sequence $\left\{X_{t}\right\}$ converges a.s. to the set $B$ as $t \rightarrow \infty$, i.e. $\rho\left(X_{t}, B\right) \rightarrow 0$ a.s. as $t \rightarrow \infty$. [Here $\rho(y, Y)$ is the Euclidean distance in $R^{1}$ from the point $y$ to the set $Y$.]

Because in the studies of urn processes [Arthur et al. (1987a, b, 1988), Hill et al. (1980)] one does not require continuity of the function $f(\cdot)$, then the set $B$ has to be properly defined. Put

$$
B=\{x \in[0,1]:[\underline{a}(x), \bar{a}(x)] \ni 0\},
$$

where $\underline{a}(x)=\liminf _{y \rightarrow x}[f(y)-y]$ and $\bar{a}(x)=\limsup _{y \rightarrow x}[f(y)-y]$. Here $y$ belongs to $R(0,1)$.

It is easy to see that if all of the connected components of $B$ are singleton, then the convergence to $B$ implies convergence of the sequence $\left\{X_{t}\right\}$. As was shown in Arthur et al. (1987b) and Hill et al. (1980), if the set

$$
\begin{aligned}
G= & \left\{x \in B: \forall \varepsilon>0 \exists y_{\varepsilon}^{-}, y_{\varepsilon}^{+} \in R(0,1) \text {, which belong to }(x-\varepsilon, x)\right. \text { or } \\
& \left.(x, x+\varepsilon), \text { such that } f\left(y_{\varepsilon}^{-}\right)<y_{\varepsilon}^{-} \text {and } f\left(y_{\varepsilon}^{+}\right)>y_{\varepsilon}^{+}\right\}
\end{aligned}
$$

is nowhere dense, then the sequence $\left\{X_{t}\right\}$ turns out to be a.s. convergent even in the case when $B$ may contain intervals.

An isolated point $\theta \in B$ is called stable if for every small enough $\varepsilon_{1}>0$ and $\varepsilon_{2}>0$

$$
[f(x)-x](x-\theta)<\delta\left(\varepsilon_{1}, \varepsilon_{2}\right)<0,
$$


provided that $\varepsilon_{1} \leqq|x-\theta| \leqq \varepsilon_{2}(x \in R(0,1))$. We shall say that an isolated point $\theta \in B$ is unstable if for every small enough $\varepsilon>0$

$$
[f(x)-x](x-\theta)>0,
$$

provided that $x \in R(0,1) \cap[(\theta-\varepsilon, \theta) \cup(\theta, \theta+\varepsilon)]$.

Theorem 2. Let $\theta \in B$ be a stable point, $\theta \in(0,1)$. Also there exist $\varepsilon_{1}>0$ and $\varepsilon_{2}>0$ such that the following inequalities hold:

$$
\begin{array}{ll}
f(x)>0 \quad \text { for } & x \in R(0,1) \cap\left(\theta-\varepsilon_{1}, \theta\right), \\
f(x)<1 \quad \text { for } \quad x \in R(0,1) \cap\left(\theta, \theta+\varepsilon_{2}\right) .
\end{array}
$$

Then $P\left\{\lim _{t \rightarrow \infty} X_{t}=\theta\right\}>0$ for every $X_{1} \in\left(\theta-\varepsilon_{1}, \theta+\varepsilon_{2}\right)$.

The proof of the theorem is essentially similar to that given in Arthur et al. (1988) and in Hill et al. (1980). We shall only note that the requirements on $f(\cdot)$ allow to shift to the left for $X_{1}>\theta$ and to the right for $X_{1}<\theta$ inside $\left(\theta-\varepsilon_{1}, \theta+\varepsilon_{2}\right)$ with positive probability through a corresponding finite number of steps.

Theorem 3. Suppose that $\theta \in B$ is an unstable point, $\theta \in(0,1)$ and one of the following conditions holds true:

(1) into a neighborhood of $\theta$ the function $f(\cdot)$ is continuous;

(2) there exists $\varepsilon>0$ such that, for $x \in(\theta-\varepsilon, \theta+\varepsilon) \cap R(0,1)$,

$$
\begin{aligned}
& {[f(x)-x](x-\theta) \geqq \lambda|x-\theta|^{1+\mu}, f(x)[1-f(x)]} \\
& \quad \geqq \underline{c}>0, \underline{c}>\bar{c}(2 \lambda+1)^{-1},
\end{aligned}
$$

where $\mu \in[0,1], \lambda>0$ and $\bar{c}=\sup _{x \in(\theta-\varepsilon, \theta+\varepsilon) \cap R(0,1)} f(x)[1-f(x)]$;

(3) into a neighborhood of $\theta$ for $x \leqq \theta(x \geqq \theta)$ one of the conditions (1) or (2) holds true and for $x>\theta(x<\theta)$ it will be $f(x)=1(f(x)=0)$.

Then $P\left\{\lim _{t \rightarrow \infty} X_{t}=\theta\right\}=0$ for every $X_{1}$.

Remark 1. The condition (3) differs from conditions (1) and (2) because it permits that $f(x)[1-f(x)]$ equals zero in a neighborhood of $\theta$. [Indeed, condition (2) postulates positiveness of the value. In the case of condition (1) from continuity of $f(\cdot)$ at $\theta$ we have $f(\theta)[1-f(\theta)]=\theta(1-\theta)>0$ and, consequently, positive values of $f(x)[1-f(x)]$ occur in the neighborhood of $\theta$.]

Proof. The case with continuous $f(\cdot)$ was studied in Hill et al. (1980). 
Under condition (2) we can apply the results from Arthur et al. (1988). Let condition (3) hold and $f(x)=1$ for $x>\theta$. Note that

$$
\begin{aligned}
P\left\{\lim _{t \rightarrow \infty} X_{t}=\theta\right\}= & P\left\{\lim _{t \rightarrow \infty} X_{t}=\theta, X_{s} \leqq \theta, s \geqq 1\right\} \\
& +P\left\{\lim _{t \rightarrow \infty} X_{t}=\theta, X_{s}>\theta \text { for some } s \geqq 1\right\} .
\end{aligned}
$$

It is clear that the second term here equals zero. (This is because the process $X_{t}, t \geqq 1$, cannot move to the left from a point lying to the right of $\theta$.) If $f(\cdot)$ is continuous to the left of $\theta$ we put

$$
f(x)=\left\{\begin{array}{lll}
f(x) & \text { for } & x \leqq \theta \\
\min [1, x+k(x-\theta)] & \text { for } & x>\theta
\end{array}\right.
$$

where $k>1$. Otherwise

$$
f(x)=\left\{\begin{array}{lll}
f(x) & \text { for } & x \leqq \theta \\
(1+\theta) / 2 & \text { for } & x>\theta
\end{array}\right.
$$

Using the results of Arthur et al. (1988) and Hill et al. (1980), for the process $Y_{t}, t \geqq 1$, corresponding to $\tilde{f}(\cdot)$, we have

$$
P\left\{\lim _{t \rightarrow \infty} Y_{t}=\theta\right\}=0
$$

for every $Y_{1}$. Hence for $Y_{1}=X_{1}$ we obtain

$$
\begin{aligned}
& P\left\{\lim _{t \rightarrow \infty} X_{t}=\theta, X_{s} \leqq \theta, s \geqq 1\right\}=P\left\{\lim _{t \rightarrow \infty} Y_{t}=\theta, Y_{s} \leqq \theta, s \geqq 1\right\} \\
& \leqq P\left\{\lim _{t \rightarrow \infty} Y_{t}=\theta\right\}=0 .
\end{aligned}
$$

Consequently, the first term in (2) equals zero too. The case where $f(x)=0$ for $x<\theta$ can be similarly studied.

The theorem is proved. 
Conditions of convergence with positive probability to points 0 and 1 are given by the next theorem.

Theorem 4 [Arthur et al. (1983)]. If $f\left(n_{\mathrm{w}}\left(n_{\mathrm{w}}+n_{\mathrm{b}}+t\right)^{-1}\right)<1$ for $t \geqq 0$ and $\sum_{t \geqq 0} f\left(n_{\mathrm{w}}\left(n_{\mathrm{w}}+n_{\mathrm{b}}+t\right)^{-1}<\infty\right.$, then $\quad P\left\{\lim _{t \rightarrow \infty} X_{t}=0\right\}>0$. Also if $f\left(\left(n_{\mathrm{w}}+t\right)\left(n_{\mathrm{w}}+n_{\mathrm{b}}+t\right)^{-1}\right)>0$ for $t \geqq 0$ and $\quad \sum_{t \geqq 0}\left[1-f\left(\left(n_{\mathrm{w}}+\right.\right.\right.$ $\left.\left.t)\left(n_{\mathrm{w}}+n_{\mathrm{b}}+t\right)^{-1}\right)\right]<\infty$, then $P\left\{\lim _{\mathrm{t} \rightarrow \infty} X_{t}=1\right\}>0$.

The following statement gives conditions which ensure convergence of $\left\{X_{t}\right\}$ with positive probability to nondegenerate intervals in every point of which $f(x)=x$. It can be proved by an argument similar to the one presented in Arthur et al. (1988).

Theorem 5. Let $(a, b) \subseteq B, a<b$, and $f(x)=x \forall x \in(a, b) \cap R(0,1)$. Also suppose, that there exist $\varepsilon_{1}>0$ and $\varepsilon_{2}>0$ such that $f(x)>0$ for $x \in\left(a-\varepsilon_{1}, a\right) \cap$ $R(0,1)$ and $f(x)<1$ for $x \in(b, b+\varepsilon) \cap R(0,1)$. Then $P\left\{\lim _{t \rightarrow \infty} \rho\left(X_{t},(a, b)\right)=\right.$ $0\}>0$ for every $X_{1} \in\left(a-\varepsilon_{1}, b+\varepsilon_{2}\right)$. Moreover, if $X_{t}$ a.s. converges to $X_{0}$, then $P\left\{X_{0} \in(a, b)\right\}>0$ for every $X_{1} \in\left(a-\varepsilon_{1}, b+\varepsilon_{2}\right)$.

In conclusion, we shall note that for $f(\cdot)$, which depends on time, i.e., at time instant $t$, balls are added with probabilities $f_{t}\left(X_{t}\right)$ and $1-f_{t}\left(X_{t}\right)$, in such a way that $\sum_{t \geq 1} t^{-1} \alpha_{t}<\infty$, Theorems 1-5 still hold. Here $\alpha_{t}=$ $\sup _{x \in[0,1] \cap R(0,1)}\left|f_{t}(x)-f(x)\right|$.

Now we are ready to formulate the main conceptual results of the paper.

\section{Sharing a market of risk averse adopters with two new competing technologies}

On the grounds of the foregoing apparatus let us examine the dynamics of two competing technologies. Consider an adoption of a unit of the $\mathbf{A}$ technology as an addition of a white ball into an urn and an adoption of a unit of the $\mathbf{B}$ technology as an addition of a black ball. The problem can be easily put into the framework of urn schemes. Let us generalize the model introduced by Arthur et al. (1983).

Suppose that the two technologies, $\mathbf{A}$ and $\mathbf{B}$, are identical in terms of some utility measure for the adopters. However, the latter are only imperfectly informed about them so that they make their choices by asking an odd number $m \geqq 1$ of adopters who are already using the technologies. An alternative hypothesis to the same effect is that there are positive (or negative) externalities in adoption which change the returns to the user along the diffusion process, but adopters, in order to estimate them, can only sample a fixed number of users. In both cases, we assume that any new adopter will choose with probability $\alpha$ the technology used by the majority 
of the sample $m$ and with probability $1-\alpha$ the technology of the minority of them. ${ }^{2}$ [For $\alpha=0$ or $\alpha=1$ the model coincides with ones considered in Arthur et al. (1983)].

The probability of choosing $\mathbf{A}$ as a function of $x_{\mathbf{A}}$, has the following form:

and

$$
p_{\mathbf{A}}\left(x_{\mathbf{A}}\right)=\alpha x_{\mathbf{A}}+(1-\alpha)\left(1-x_{\mathbf{A}}\right)=(1-\alpha)+(2 \alpha-1) x_{\mathbf{A}} \text { for } m=1
$$

$$
\begin{aligned}
p_{\mathbf{A}}\left(x_{\mathbf{A}}\right)= & \alpha p\left(m, x_{\mathbf{A}}\right)+(1-\alpha)\left[1-p\left(m, x_{\mathbf{A}}\right)\right]+o(1)=1-\alpha \\
& +(2 \alpha-1) p\left(m, x_{\mathbf{A}}\right)+o(1) \text { for } m>1,
\end{aligned}
$$

where $p(m, x)=\sum_{i=(m+1) / 2}^{m} C_{m}^{i} x^{i}(1-x)^{m-i}\left(C_{m}^{i}\right.$ is the number of combinations form $m$ to $i$ ). Moreover, $o(1)$ goes to zero (as const. $n^{-1}$ ) uniformly on $x \in[0,1]$ for $n \rightarrow \infty$ (here $n$ designates the number of e.g. consumers who have adopted one of the technologies). The function $f(x)=1-$ $\alpha+(2 \alpha-1) p(m, x)$ for $m \geqq 1$ is given graphically in fig. 1 . Continuous lines correspond here to the case whereby $\alpha>\frac{1}{2}\left[1+\left(1 / p^{\prime}\left(m, \frac{1}{2}\right)\right)\right]$; broken lines to the case whereby $\alpha<\frac{1}{2}\left[1+\left(1 / p^{\prime}\left(m, \frac{1}{2}\right)\right)\right]$; and the horizontal line to $\alpha=1 / 2$. Using the results from section 2 the following conclusions can be drawn.

For $m=1$ and for every $\alpha<1$ the function $f(x)-x$ has the only root $x=$ 1/2. Consequently, Theorem 1 shows that $X_{t}$ (the proportion of the $\mathbf{A}$ technology on the market at time $t$ ) converges with probability 1 to $1 / 2$ as $t \rightarrow \infty$. That is, the market is shared in the limit by $\mathbf{A}$ and $\mathbf{B}$ in the proportion $1: 1$. For $m>1$ and $\alpha \leqq \frac{1}{2}\left[1+\left(1 / p^{\prime}\left(m, \frac{1}{2}\right)\right)\right]$ exactly the same argument shows that in the limit the market is shared by $\mathbf{A}$ and $\mathbf{B}$ in the proportion 1:1. For $m>1$ and $\alpha>\frac{1}{2}\left[1+\left(1 / p^{\prime}\left(m, \frac{1}{2}\right)\right)\right]$ the function $f(x)-x$ has three zeros: $x_{0}(\alpha), 1 / 2$ and $x_{1}(\alpha)=1-x_{0}(\alpha)$. Theorem 1 gives convergence of $X_{t}$ with probability 1 to the set $B=\left\{x_{0}(\alpha), 1 / 2, x_{1}(\alpha)\right\}$ as $t \rightarrow \infty$. As far as both $x_{0}(\alpha)$ and $x_{1}(\alpha)$ turn out to be stable, Theorem 2 assures that $X_{t}$ converges with positive probability to either $x_{0}(\alpha)$ or $x_{1}(\alpha)$ (from every initial approximation). Finally the observations that $1 / 2$ is an unstable point together with Theorem 3 (in the case when condition (1) occurs) show that $X_{t}$ converges to $1 / 2$ with zero probability (from every initial approximation). Consequently, for $m>1$ and $\alpha>\frac{1}{2}\left[1+\left(1 / p^{\prime}\left(m, \frac{1}{2}\right)\right)\right]$, in the limit, the market can be shared by $\mathbf{A}$ and $\mathbf{B}$ in proportions (each with positive probability) $x_{0}(\alpha):\left[1-x_{0}(\alpha)\right]$ and $\left[1-x_{0}(\alpha)\right]: x_{0}(\alpha)$.

The foregoing argument shows how the results of section 2 can be used to describe limit states for a given urn function. Let us just mention some further properties which follow from the theorems of section 2 .

\footnotetext{
${ }^{2}$ Note that the model is agnostic with respect to specific theories of learning and choice, but consistent with a few of them. For example, one could assume that $\alpha \geqq \frac{1}{2}\left[1+\left(1 / p^{\prime}\left(m, \frac{1}{2}\right)\right)\right]$ and increases with the sample size. (That is, agents weight their observations by some statistical criterion of reliability.) At the opposite extreme, the model may easily accommodate behaviors such as the search for diversity in consumption ('I don't like what the majority likes...'). Finally, the model can account for the persistence of non-average behaviors [on financial markets, cf. Kirman (1991)].
} 


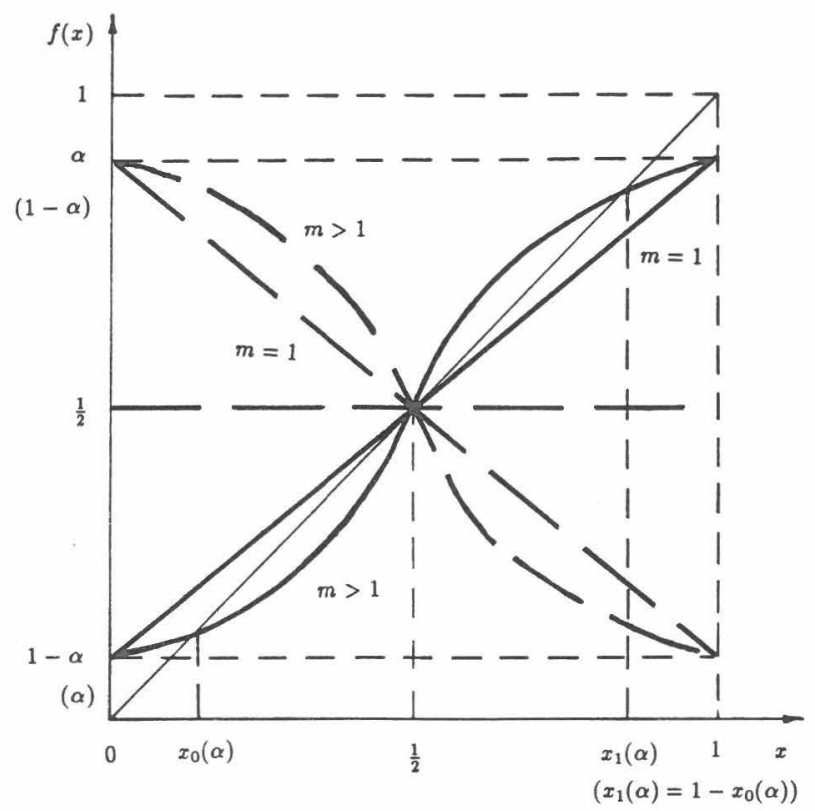

Fig. 1

The main new elements of this model compared with those obtained in Arthur et al. (1983) are the following:

(a) if $m=1$, then for every $\alpha<1$ there is only one feasible limit market sharing, in contrast with the case $\alpha=1$, where, firstly, feasible limit market shares coincide with the whole closed interval $[0,1]$ and, secondly, these limit market shares belong to each of subintervals $(a, b) \in[0,1]$ with positive probability;

(b) if $m>1$, then for every $\alpha<1$, unlike the case $\alpha=1$, there is no monopoly market shares [because $x_{0}(\alpha)>0$ and consequently $x_{1}(\alpha)<1$ ] in spite of the fact that $x_{0}(\alpha) \rightarrow 0$ and, thus, $x_{1}(\alpha) \rightarrow 1$ as $\alpha \rightarrow 1$.

From an economic point of view, the result shows, it is an 'imperfect' process of information-acquisition (or endogenous preference formation) which curbs the tendency toward technological monopoly and allows an equilibrium co-existence of variety. (Note also that this variety may simply be based on equilibrium distributions of diverse expectations on otherwise identical technologies, in terms of utility derived from them. $)^{3}$

\footnotetext{
${ }^{3}$ Clearly, the applicability of the model is by no means restricted to technical change: for example, it could be used to interpret estimates of the 'fundamentals' in macroeconomic models and the ensuing coexistence of diverse 'models of the world'.
} 


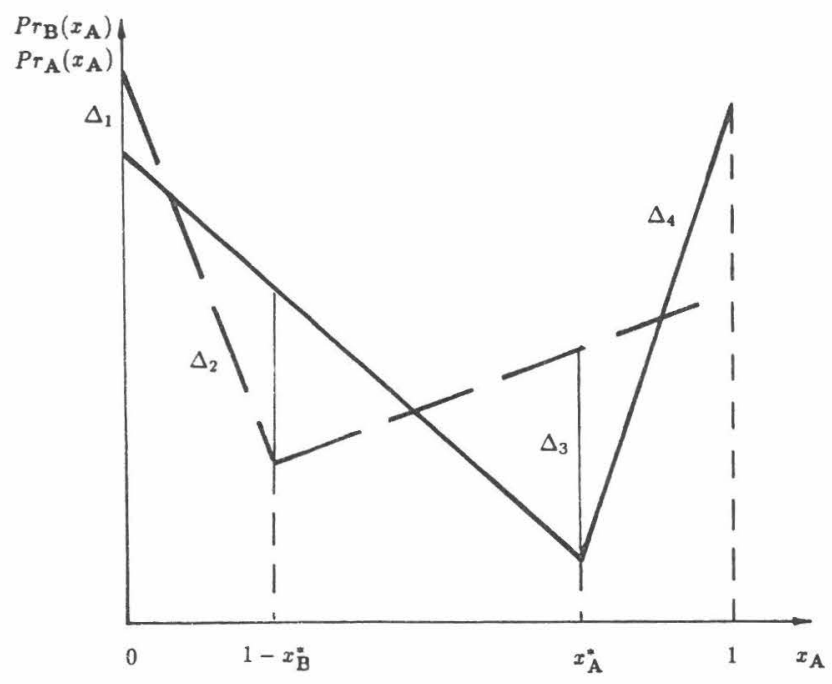

Fig. 2

\section{A model of competition under implicit preferences of consumers in the case of approximately equal prices}

Let us now introduce a price dynamics for the two technologies. Suppose, that two firms compete for a market of infinite capacity. Let us now designate both the firms and their products (technologies) with $\mathbf{A}$ and $\mathbf{B}$. As a first approximation to a pricing strategy, consider the following: each firm reduces the price until it reaches a certain level of market share, defined by the proportion of the product of this firm among all products which have been sold up to the current time (usually greater than 1/2). Above that level it increases it. Let us consider the simplest (linear) case of this policy. This is graphically represented in fig. 2. Here $\operatorname{Pr}_{\mathrm{A}}\left(x_{\mathrm{A}}\right)$ designates the dependence of the price of the technology $\mathbf{A}$ as a function of its proportion $x_{\mathrm{A}}$ among all adopters who are using either technology. Symmetrically, $\operatorname{Pr}_{\mathbf{B}}\left(x_{\mathbf{A}}\right)$ designates the dependence of the price of the technology $\mathbf{B}$ as a function of $x_{\mathbf{A}}$. [Of course, the proportions of technologies $\mathbf{A}$ and $\mathbf{B}$ are complements: $x_{\mathbf{A}}+x_{\mathbf{B}}=$ 1.] By $x_{\mathbf{A}}^{*}$ and $x_{\mathbf{B}}^{*}$ we designate the critical levels of market shares which switch from falling- to rising-price rules. Hence, the dependence of the price of the $\mathbf{A}(\mathbf{B})$ technology on its proportion on the market $x_{\mathbf{A}}\left(x_{\mathbf{B}}\right)$ is given by four parameters: $\operatorname{Pr}_{\mathbf{A}}(0), x_{\mathrm{A}}^{*}, \operatorname{Pr}_{\mathbf{A}}\left(x_{\mathrm{A}}^{*}\right), \operatorname{Pr}_{\mathbf{A}}(1)\left[\operatorname{Pr}_{\mathbf{B}}(1), x_{\mathrm{B}}^{*}, \operatorname{Pr}_{\mathrm{B}}\left(1-x_{\mathrm{B}}^{*}\right), \operatorname{Pr}_{\mathbf{B}}(0)\right]$. Note that the model accounts also for the circumstances where $\operatorname{Pr}_{\mathbf{A}}(1) \leqq$ $\operatorname{Pr}_{\mathbf{A}}\left(x_{\mathbf{A}}^{*}\right)\left[\operatorname{Pr}_{\mathbf{B}}(0) \leqq \operatorname{Pr}_{\mathbf{B}}\left(1-x_{\mathbf{B}}^{*}\right)\right]$, such as when $x_{\mathbf{A}}^{*}=1\left(x_{\mathbf{B}}^{*}=1\right)$ : in this case, firm $\mathbf{A}$ (B) reduces indefinitely the price on its product as its market share increases.

It is natural to suppose that in the case where the 'quality' of the 
technologies is approximately the same and potential consumers know about it, the technology which is cheaper has more chances of being sold, i.e., the $\mathbf{A}$ technology is bought if $\operatorname{Pr}_{\mathbf{A}}\left(x_{\mathrm{A}}\right)-\operatorname{Pr}_{\mathbf{B}}\left(x_{\mathrm{A}}\right)<0$. But, if the prices differ slightly or consumers have some specific preferences (which can be characterized only statistically or on average), this sometimes leads to the adoption of the more expensive technology. Such a situation can be mathematically formalized in the following way [see also Hanson (1985)]. The A technology is bought if $\operatorname{Pr}_{\mathbf{A}}\left(x_{\mathbf{A}}\right)-\operatorname{Pr}_{\mathbf{B}}\left(x_{\mathbf{A}}\right)+\xi<0$, where $\xi$ is a random variable. Then the probability $f\left(x_{\mathrm{A}}\right)$ to choose the $\mathbf{A}$ technology, as a function of $x_{\mathbf{A}}$, equal $P\left\{\xi<\operatorname{Pr}_{\mathbf{B}}\left(x_{\mathbf{A}}\right)-\operatorname{Pr}_{\mathbf{A}}\left(x_{\mathbf{A}}\right)\right\}$. To avoid unnecessary sophistications of the model, we shall suppose that $\xi$ possesses density with respect to the Lebesgue measure in $R^{1}$ [otherwise the event ' $\operatorname{Pr}_{\mathrm{B}}\left(x_{\mathrm{A}}\right)-\operatorname{Pr}_{\mathrm{A}}\left(x_{\mathrm{A}}\right)+\xi=0$ ' can have positive probability]. Also, it is natural to suppose that $\xi$ has a bounded support. This means that $P\{\xi \in[-\alpha, \alpha]\}=1$ for some $\alpha>0$. That is, adopters have a 'threshold' decision rule: above a certain price differential they choose deterministically; below, they follow randomized strategies. To simplify our considerations, suppose that $\xi$ has a uniform distribution on $[-\alpha, \alpha]$. Since random factors appear when prices on $\mathbf{A}$ and $\mathbf{B}$ are approximately equal, then the inequality $\alpha<\min _{i=1,2,3,4} \Delta_{i}$ holds. The probability of choosing $\mathbf{A}$ as a function of $x_{\mathrm{A}}$ in this case has the form

$$
f\left(x_{\mathbf{A}}\right)= \begin{cases}1 & \text { for } \operatorname{Pr}_{\mathbf{B}}\left(x_{\mathbf{A}}\right)-\operatorname{Pr}_{\mathbf{A}}\left(x_{\mathbf{A}}\right) \geqq \alpha, \\ 0 & \text { for } \operatorname{Pr}_{\mathbf{B}}\left(x_{\mathbf{A}}\right)-\operatorname{Pr}_{\mathbf{A}}\left(x_{\mathbf{A}}\right) \leqq-\alpha,(3) \\ {\left[\operatorname{Pr}_{\mathbf{B}}\left(x_{\mathbf{A}}\right)-\operatorname{Pr}_{\mathbf{A}}\left(x_{\mathbf{A}}\right)+\alpha\right] / 2 \alpha} & \text { for } \quad-\alpha<\operatorname{Pr}_{\mathbf{B}}\left(x_{\mathbf{A}}\right)-\operatorname{Pr}_{\mathbf{A}}\left(x_{\mathbf{A}}\right)<\alpha .\end{cases}
$$

This is graphically represented in fig. 3. Hence, the model embodies a positive feedback mechanism of diffusion: prices fall with increasing market shares possibly due to learning economies, dynamic increasing returns, etc., and/or, on the behavioral side, to market-penetration strategies. However, the mechanism is bounded: above a certain market share, the price starts to rise, possibly due to monopolistic behaviors of the producer(s) and/or to the progressive exhaustion of technological opportunities to lower production costs. Finally, market adjustments as a function of differential prices are 'imperfect': within boundaries, differently priced technologies both face positive demand. What can one say on the limit shares of such a boundedincreasing-returns process of diffusion? Using the results of section 2 we have the following:

(1) convergence to $x_{1}^{*}$ with probability 1 takes place from the domain I (cf. fig. 3);

(2) from the domain II there is convergence with positive probability to both $x_{1}^{*}$ and $x_{3}^{*}$; 


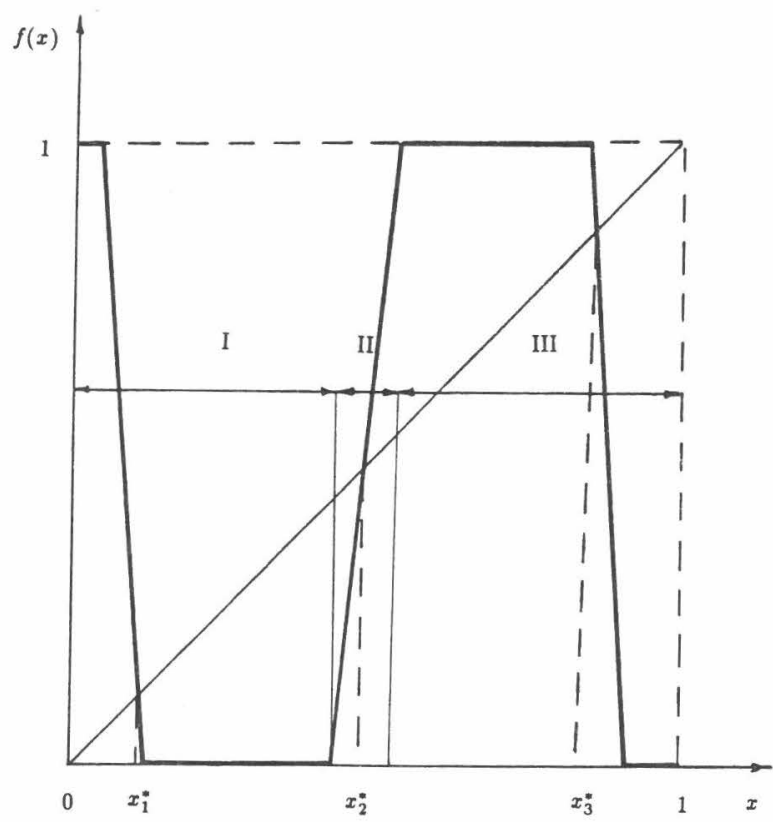

Fig. 3

(3) convergence to $x_{3}^{*}$ with probability 1 takes place from the domain III.

In terms of competing technologies, these results can be conceptually treated in the following way. If the initial proportion of adopters of technology A belongs to the domain I (III), then the technologies A and B share the market in the proportion $x_{1}^{*}:\left(1-x_{1}^{*}\right)\left(x_{3}^{*}:\left(1-x_{3}^{*}\right)\right)$. Moreover, if the initial proportion of adopters of technology $\mathbf{A}$ belongs to the domain II, then $\mathbf{A}$ and $\mathbf{B}$ share the market in the proportion $x_{1}^{*}:\left(1-x_{1}^{*}\right)$ or $x_{3}^{*}:\left(1-x_{3}^{*}\right)$ (with positive probability in each of the cases).

More generally: diffusion with endogenous prices and bounded dynamic increasing returns yields market-sharing rather than monopoly. Still, limit market shares are path-dependent: they are determined by the history of the diffusion process. The model, however, allows a qualitative analysis - by no means restricted to the price dynamics assumed here - of the ensuing limit proportions dependent on the relative frequencies of initial adopters of the different technologies.

Let us now turn to the effects on diffusion dynamics of different degrees of 'market stickiness', as approximated by $\alpha$.

If $\alpha=\Delta_{i}$ for some $i$, then the corresponding horizontal part of the graph of $f(\cdot)$ converts into a 'sharp', where $f(\cdot)$ attains 0 or 1 . If the distribution of $\xi$ is not uniform, then sloping (straight) line segments of the graph of $f(\cdot)$ 


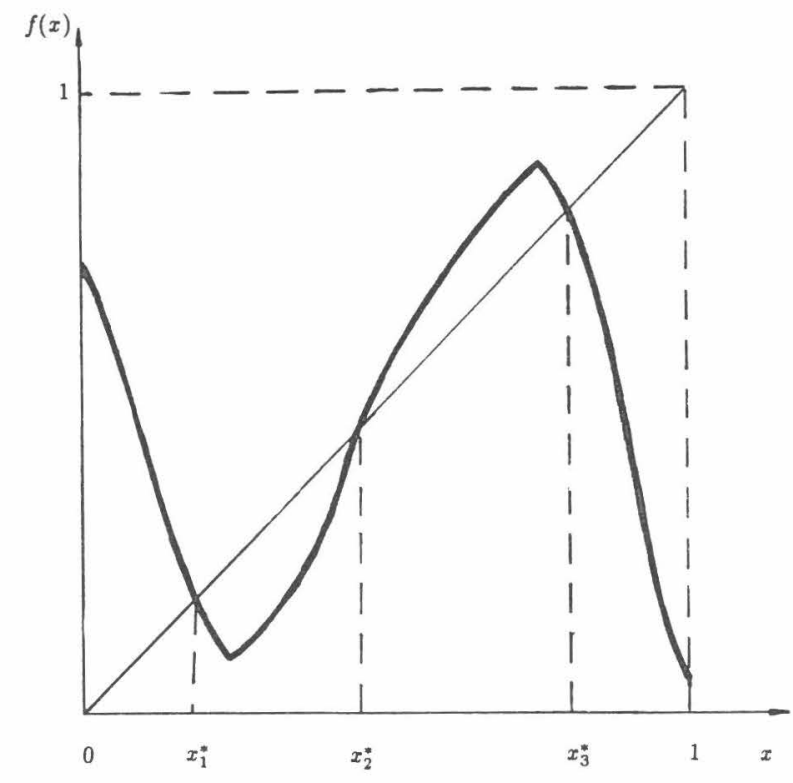

Fig. 4

convert into curve linear ones. And, finally, if $\alpha>\max _{i=1,2,3,4} \Delta_{i}$ (in particular, when $\xi$ has an unbounded support, as in the normal distribution), then all of horizontal segments transform into 'sharps' of corresponding height [from $(0,1)]$. In this case we can obtain the graph presented in fig. 4 . Here [as follows from the results of Arthur et al. (1987a, 1988), Hill et al. (1980)], convergence with positive probability (from every initial approximation) takes place to both $x_{1}^{*}$ and $x_{3}^{*}$. Consequently, regardless of the initial proportions of adopters of the two technologies, $\mathbf{A}$ and $\mathbf{B}$ share the market in the proportions $x_{1}^{*}:\left(1-x_{1}^{*}\right)$ or $x_{3}^{*}:\left(1-x_{3}^{*}\right)$. Which one depends on chance.

Implicit preferences of adopters (or, which is basically the same, preferences with imperfect information and 'market-stickiness') can be formalized in a slighly different way. Suppose that if the difference of the prices is not less than $\alpha>0$, then the cheaper technology is bought. If the difference is less than the value, then consumers to choose a technology use some stochastic experiment (i.e., a mixed strategy). Here $\alpha<\min _{i=1,2,3,4} \Delta_{i}$. Consider the following examples:

Case 1. A is chosen with probability $p \in(0,1)$ and $\mathbf{B}$ with probability $1-p$ (usually, when there is no a priori preference, $p=1 / 2$ ).

Case 2. Would-be adopters sample an odd number $m>1$ of adopters who have already selected one of the technologies and choose the technology which is used by the majority (minority) of them. 


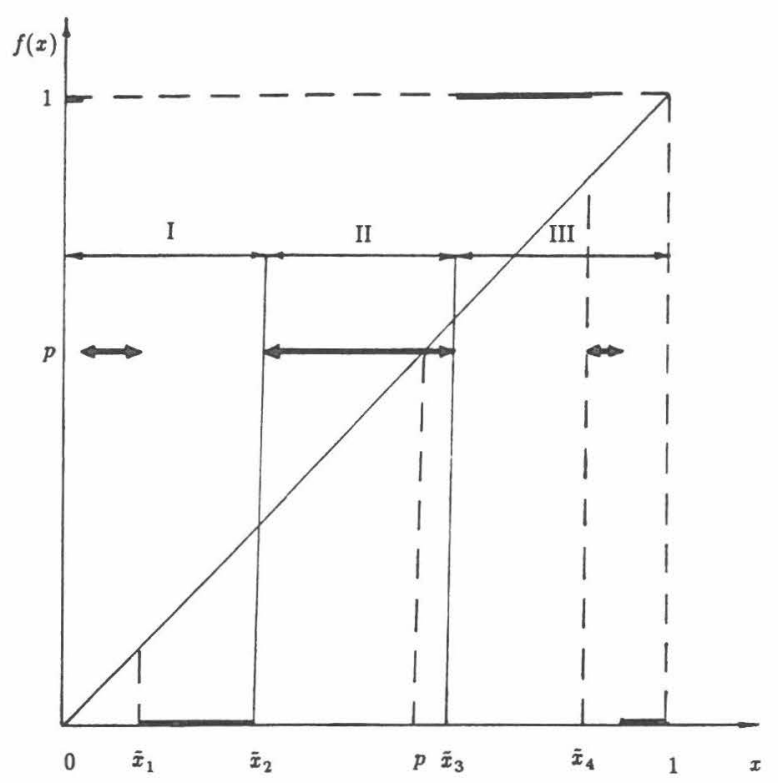

Fig. 5

The corresponding probabilities $f\left(x_{\mathbf{A}}\right)$ to choose $\mathbf{A}$ as a function of $x_{\mathrm{A}}$, are represented graphically in figs. 5 and 6 (in the latter case we neglect the term which goes to zero; see section 3). Note that in fig. 6 in order to designate those parts of the graph where $f(\cdot)$ does not attain either 0 or 1 , we use continuous (broken) lines according to whether the choice follows the majority (minority) of the sample.

Using the results from section 2, in case 1, we obtain: (i) from the domain I convergence to $x_{1}^{*}$ with probability 1; (ii) convergence with positive probability to $x_{1}^{*}, p$ and $x_{4}^{*}$ from the domain II; (iii) convergence to $x_{4}^{*}$ with probability 1 from the domain III. Hence, the limit market-shares properties are similar to those considered earlier in this section.

However, consider now the case of endogenous prices, as above, jointly with endogenous preferences for either technology, whereby choices corresponds to the option of the sampled majority. We obtain the following limit shares: (1) from the domain I convergence to $x_{1}^{*}$ with probability 1 ; (2) convergence with positive probability to both $x_{1}^{*}$ and $x_{2}^{*}$ from the domain II; (3) from the domain III convergence with probability 1 to $x_{2}^{*}$.

In all of the considered cases, monopoly (i.e., the situation where one of the technologies conquers the market) is impossible. To reach monopoly one must change the relation between prices on $\mathbf{A}$ and $\mathbf{B}$. As it might be intuitive if systematically $\operatorname{Pr}_{\mathbf{A}}(0)>\operatorname{Pr}_{\mathbf{B}}(0)$, then we can have monopoly of the $\mathbf{B}$ 


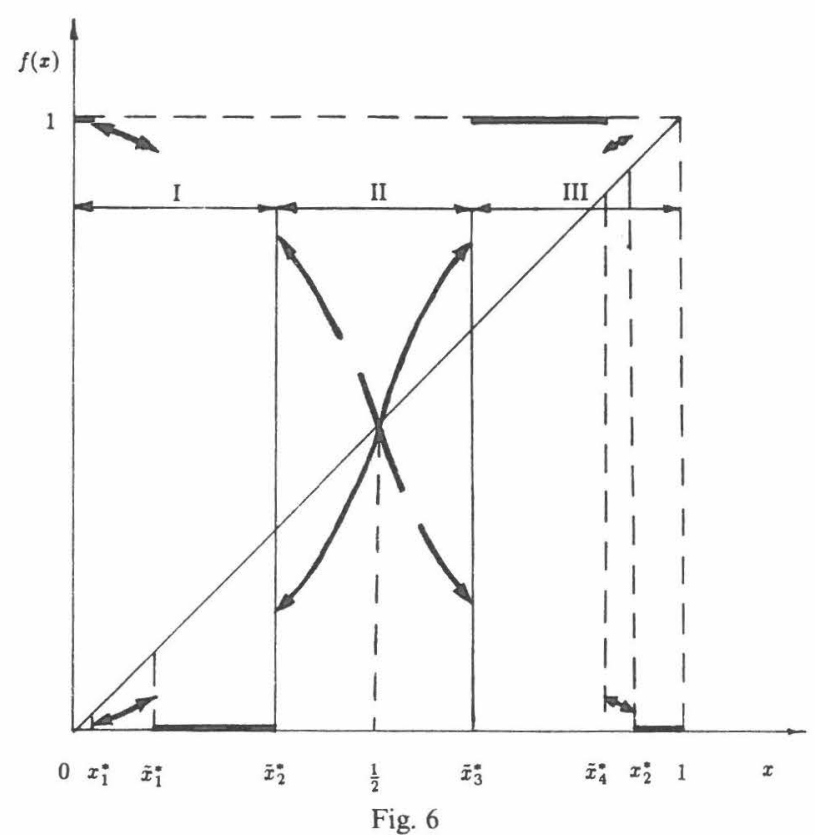

technology. Conversely, if $\operatorname{Pr}_{\mathbf{A}}(1)<\operatorname{Pr}_{\mathbf{B}}(1)$, one can arrive at monopoly of technology A.

More generally, though, the model highlights the crucial importance of specific price dynamics in the determination of limit market shares. Some (more 'evolutionary' inclined) economists might interpret the result as an analytical corroboration of the conjecture that out-of-equilibrium 'boundedly rational' behavioral norms do affect system-level asymptotic states. ${ }^{4}$ Alternatively one may argue that all this simply emphasizes the dependence of limit market shares upon expectations and intertemporal discount procedures of supposedly perfect rational but imperfectly informed producers of each technology. ${ }^{5}$ Irrespective of the precise microeconomic assumption, again, the model allows qualitative analyses of the relationships between endogenous price changes, adoption frequencies and limit market shares.

By way of illustration, suppose for example that the switching point between price-decreasing and price-increasing strategies occurs at less than $1 / 2$ market shares. In this case, we obtain the picture shown in fig. 7. Moreover, if price-dependent choices involve a random error uniformly

\footnotetext{
${ }^{4}$ In such an evolutionary perspective, pricing 'strategies' may be understood as routines setting desired profit margins as a function of asymmetries in production efficiency or in the ability to exploit market power: cf. Nelson and Winter (1982) and Dosi (1984).

${ }^{5}$ Yet another hypothesis is that the price dynamics assumed above is some crude approximation to a Bertrand game under (bounded) increasing returns.
} 


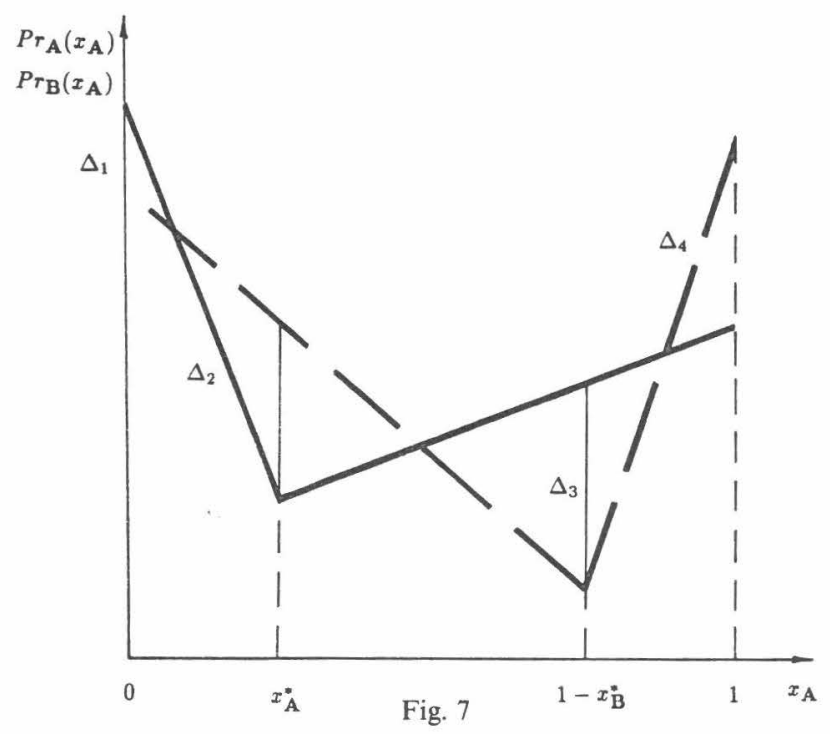

distributed on $[-\alpha, \alpha]$ (with $\alpha<\min _{i=1,2,3,4} \Delta_{i}$ ), then the probability of choosing $\mathbf{A}$ as a function of $x_{\mathbf{A}}$ is illustrated in fig. 8. Using the results from section 2, we have: (1) if the initial proportion of adopters of the technology A belongs to the domain I (V), then B (A) conquers the market; (2) if the proportion belongs to the domain III, then $\mathbf{A}$ and $\mathbf{B}$ share the market in the proportion $x_{2}^{*}:\left(1-x_{2}^{*}\right)$; (3) if the initial proportion of adopters of the technology A belongs to the domain II (IV), then the technology B (A) conquers market shares in the proportion $x_{2}^{*}$ : $\left(1-x_{2}^{*}\right)$.

Consequently, under the given hypotheses concerning the behavior of adopters, massive introduction of one of the technologies (domains I and V), which is significantly cheaper in these domains, leads to monopoly. Under less massive introduction (domains II and IV) we can either have monopoly or alternatively, $\mathbf{A}$ and $\mathbf{B}$ share the market in the proportion $x_{2}^{*}$ : $\left(1-x_{2}^{*}\right)$. In the case of comparable initial numbers of adopters of $\mathbf{A}$ and $\mathbf{B}$ (domain III) these technologies share the market in the proportion $x_{2}^{*}:\left(1-x_{2}^{*}\right)$.

Note also that the formal apparatus developed here can be used to study all cases whereby prices depend on the current concentration of one of the technologies on the market in an arbitrary way (while here for the sake of simplicity we restricted the discussion to cases where these functions are piecewise linear).

\section{Conclusions}

Innovation and technology diffusion generally involve competition among 


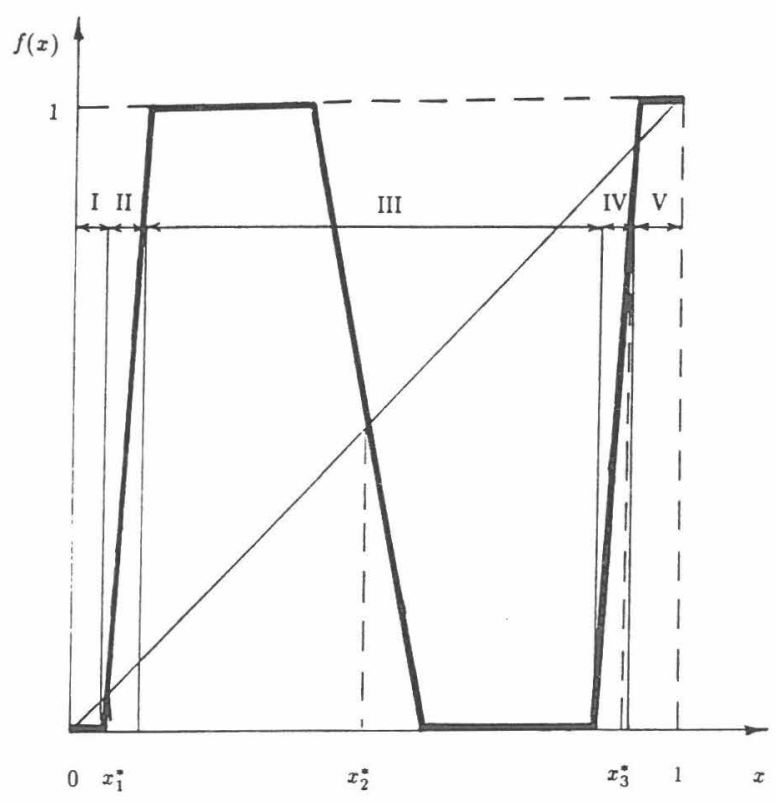

Fig. 8

different technologies, and, most often, endogenous changes in the costs/ prices of technologies themselves and in adopters' choices. In the economic domain (as well as in other disciplines) the formal representation of such processes involves some dynamics of competing 'populations' (i.e., technologives, firms, or even behavioral traits and 'models' of expectation formation). A growing literature on such dynamics has begun studying the properties of those (generally non-linear) processes that innovation and diffusion entail. As is now robustly established, multiple equilibria are normally to be expected. 'History matters', also in the sense that out-of-equilibrium fluctuations may bear system-level consequences on notional asymptotic outcomes. Developing on previous results showing - under dynamic increasing returns - the likely 'lock-in' of diffusion trajectories onto particular technologies, we have presented a formal modeling apparatus aimed at handling the interaction between diffusion patterns, on the one hand, and endogenous preferences formation and/or endogenous price formation, on the other. As examples, we presented two stochastic models of shares dynamics on a market of infinite capacity by two competing new technologies. In the first of them, we assumed that the adoption dynamics is essentially driven by endogenous changes in the choices of risk-averse, imperfectly informed adopters (or, in a formally equivalent analogy, by some positive or negative externality imper- 
fectly estimated by would-be users of alternative technologies). In the second example, we considered an endogenous price dynamics of two alternative technologies, driven by e.g., changes in their costs of production and/or by the intertemporal behaviors of their producers.

In both cases, the diffusion process is allowed to embody some stochasticity, due to e.g., 'imperfect' learning from other people's choices, marginal and formally undetectable differences in users' preferences, or some inertia in adjusting between differently priced but identical-return technologies.

The formal apparatus presented here, based on a few refinements on generalized urn schemes, allows quite general analytical accounts of the relationships between some system-parameters (e.g., proxies for information 'imperfection' by adopters; dynamic increasing returns and monopolistic exploitation of new technologies by their producers) and limit market shares. While path-dependency (i.e. 'history matters') applies throughout, the foregoing analytical techniques appear to be able, at the very least, to discriminate those which turn out to be feasible limit equilibria (i.e., those which are attainable with positive probabilities) and, also, to 'map' them into the relative frequencies of initial adopters.

As the foregoing modeling illustrations show, 'market imperfections' and 'informational imperfections' often tend to foster technological variety, i.e., the equilibrium co-existence of different technologies and firms. Moreover, stochasticity in the choice process may well bifurcate limit market-shares outcomes. Finally, we have shown that corporate pricing strategies - possibly based on boundedly rational procedures, imperfect infomation or even systematically 'wrong' expectation-formation mechanisms - generally influence long-term outcomes. Under all these circumstances, the foregoing modeling techniques allow, at the very least, a 'qualitative' analytical assessment of diffusion/competition processes by no means restricted to those cases where microeconomic expectations, on average, represent unbiased estimations of the future.

Whenever this analytical representation is empirically adequate, there seem to be no a priori reasons to restrict it to technological dynamics. In fact, under suitable modifications, it may apply as well to interdependent expectations, decision and returns on e.g., industrial or financial markets. Ultimately, what we have tried to implement is a relatively general analytical apparatus able to handle at least some qualitative properties of dynamic stochastic processes characterized by both positive, and, possibly, negative, feedbacks of a functional form as 'badly-behaved' as possible. Indeed, we believe, quite a few of the processes of economic change fall into this category, related to technological change but also to interdependent (possibly 'disequilibrium') changes in e.g., industrial structures, financial markets or macroeconomics dynamics. 


\section{References}

Anderson, P.W., K.F. Arrow and R. Pines, eds., 1988, The economy as an evolving complex system (Addison-Wesley, New York).

Arthur, W.B., 1988, Self-reinforcing mechanisms in economics, in: P.W. Anderson, K.F. Arrow and R. Pines, eds., The economy as an evolving complex system (Addison-Wesley, New York) 9-31.

Arthur, W.B. and D.A. Lane, 1993, Information constriction and information contagion, Structural Change and Economic Dynamics, forthcoming.

Arthur, W.B.. Yu.M. Ermoliev and Yu.M. Kaniovski, 1983, The generalized urn problem and its application, Kibernetika 1, 49-56 (in Russian).

Arthur, W.B., Yu.M. Ermoliev and Yu.M. Kaniovski, 1987a, Adaptive process of growth being modeled by urn schemes, Kibernetika 6, 49-57 (in Russian).

Arthur, W.B., Yu.M. Ermoliev and Yu.M. Kaniovski, 1987b, Non-linear urn processes: Asymptotic and applications, WP-87-85 (International Institute for Applied Systems Analysis, Laxenburg, Austria).

Arthur, W.B., Yu.M. Ermoliev and Yu.M. Kaniovski, 1987c, Path dependent processes and the emergence of macro-structure, European Journal of Operational Research 30, no. 1, 294-303.

Arthur, W.B., Yu.M. Ermoliev and Yu.M. Kaniovski, 1988, Non-linear adaptive processes of growth with general increments: Attainable and unattainable components of terminal set, WP-88-86 (International Institute for Applied Systems Analysis, Laxenburg, Austria).

David, P.A., 1985, Clio and the economics of QWERTY, American Economic Review, Papers and Proceedings 75, 332-337.

David, P.A., 1993, Putting the past into the future of economics, Journal of Economic Literature, forthcoming.

Dosi, G., 1984, Technical change and industrial transformation (McMillan, London and St. Martin Press, New York).

Dosi, G., 1988, Sources, procedures and microeconomic effects of innovation, Journal of Economic Literature, 1120-1171.

Dosi, G., C. Freeman, R. Nelson, G. Silverberg and L. Soete, eds., 1988, Technical change and economic theory (Francis Pinter, London).

Frydman, R., 1982, Towards an understanding of market processes: Individual expectations, learning and convergence to rational expectations equilibrium. American Economic Review $72,652-668$.

Frydman, R. and E.S. Phelps, eds., 1983, Individual forecasting and aggregate outcomes (Oxford University Press, Oxford).

Glaziev, S.Yu. and Yu.M. Kaniovski, 1991, Diffusion of innovations under conditions of uncertainty: A stochastic approach, in: N. Nakićenović and A. Grübler, eds., Diffusion of technologies and social behavior (Springer-Verlag, Berlin, Heidelberg, New York) 231-246.

Hanson, W.A., 1985, Bandwagons and orphans: Dynamic pricing of competing systems subject to decreasing costs. Ph.D. dissertation (Stanford University, Stanford, CA).

Hill, B.M., D. Lane and W. Sudderth, 1980, A strong law for some generalized urn processes, The Annals of Probability 8, no. 2, 214-226.

Kirman, A.P., 1991, Epidemics of opinion and speculative bubbles in financial markets, in: M. Taylor, ed., Money and financial markets (Macmillan, London).

Jordan, J., 1985, Learning rational expectation: The finite state case, Journal of Economic Theory $36,257-276$.

Nelson, R.R. and S. Winter, 1982, An evolutionary theory of economic change (The Belknap Press of Harvard University Press, Cambridge, MA).

Polterovich, V.M. and G.M. Henkin, 1988, An economic model interaction of processes of creation and leasing of technologies, Ekonomika i Matematicheskie Metody 24, no. 6, 10711083 (in Russian).

Silverberg, G., G. Dosi and L. Orsenigo, 1988, Innovation, diversity and diffusion: A selforganization model, The Economic Journal 98, 1032-1054. 
